\title{
Dosis elevada en el dosímetro personal de una auxiliar de enfermería en Electrofisiología
}

\section{A high dose in the personal dosage meter of nursing assistant in Electrophysiology field}

\section{Raquel Barquero Sanz'1, Jerónimo Jesús Rubio Sanz², Fernando Rescalvo Santiago³}

1 Servicio de Protección Radiológica. Hospital Clínico Universitario de Valladolid. Valladolid. España.

2 Servicio de Cardiología. Hospital Clínico Universitario de Valladolid. Valladolid. España.

3 Servicio de Prevención de Riesgos Laborales. Hospital Clínico Universitario de Valladolid. Valladolid. España.

Recibido: 13-12-11

Aceptado: 10-01-12

\section{Correspondencia}

Raquel Barquero Sanz

Servicio de Protección Radiológica

Hospital Clínico Universitario de Valladolid

Avda. Ramón y Cajal, 3

47005 Valladolid. España

Tfno: 983420000

E-mail: rbarquerosa@saludcastillayleon.es

\section{Resumen}

En una sala de Electrofisiología del Servicio de Cardiología en donde se efectúan procedimientos guiados con rayos $\mathrm{X}$ de ablación con radiofrecuencia y de implantes de desfibrilador biventricular, se registraron dosis mensuales anormalmente elevadas en el dosímetro personal de solapa de una auxiliar de enfermería que trabaja en la misma, con valores de dosis equivalente personal, $\mathrm{Hp}(10)$, de $125 \mathrm{mSv}$ y de dosis equivalente personal superficial, $\mathrm{Hp}(0.07)$ de $128.1 \mathrm{mSv}$.

Ante la ansiedad producida en la trabajadora al conocer las lecturas de su dosímetro, y dado que no se dispone de precedentes investigados en la instalación, se ha realizado un estudio para dilucidar si la dosis registrada ha podido ser recibida por la trabajadora o si solo ha sido recibida por el dosímetro.

Se hacen medidas con un monitor ambiental en condiciones simuladas de los procedimientos, para estimar las dosis reales que pueda recibir el personal de la sala. Se comparan estos valores con los del rendimiento del equipo de $\mathrm{RX}$ en haz directo medidos durante la revisión anual de control de calidad del mismo, concluyéndose que ha sido el dosímetro, y no la trabajadora, el que se ha expuesto a radiación directa, siendo la situación más probable la de que dicho dosímetro cayó sobre el tubo de RX, normalmente orientado hacia el techo, un tiempo del orden de 1 minuto.

Paralelamente a estas estimaciones dosimétricas, el Servicio de Prevención de Riesgos Laborales realizó la vigilancia de la salud, concluyendo que la trabajadora no tenía ningún indicio de haber recibido dosis de la magnitud registrada por su dosímetro.

Med Segur Trab (Internet) 2012; 58 (226) 59-66

Palabras clave: Dosimetría, exposición accidental, radiaciones ionizantes. 


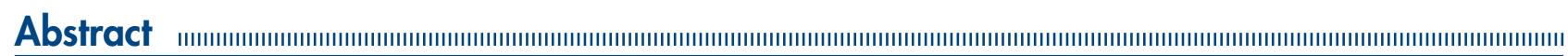

In a room of electrophysiology from the cardiology service where procedures are made guided with X-Rays of ablation with radiofrequency and from implants ventricular defibrillation; there were registered monthly doses usually high in the same personal dosimeter flap of a nursing assistant who works there with values of equivalent personal doses, Hp (10), of superficial equivalent doses, Hp (0.07) of 128.1mSv.

In view of the anxiety produced in the worker upon knowing the readings of its dosimeter, and since there is no disposition of precedents investigated in the facility, and it has been carried out a study for explaining if the doses registered could has been received by the worker if it is only received by the dosimeter.

Med Segur Trab (Internet) 2012; 58 (226) 59-66

Key words: Dosimetry, accidental exposure, ionizing radiaton. 


\section{INTRODUCCIÓN}

En una sala de Electrofisiología del Servicio de Cardiología en donde se efectúan procedimientos guiados con rayos $\mathrm{X}$ de ablación con radiofrecuencia (radiofrequency catheter ablation RFCA) y de implantes de desfibrilador biventricular (biventricular internal cardioverter defibrillator BiVICD), se registraron dosis mensuales anormalmente elevadas en el dosímetro personal de solapa de una auxiliar de enfermería que trabaja en la misma, con valores de dosis equivalente personal, $\mathrm{Hp}(10)$, de $125 \mathrm{mSv}$ y de dosis superficial $\mathrm{Hp}(0.07)$ de $128.1 \mathrm{mSv}$.

Ante la ansiedad producida en la trabajadora al conocer las lecturas de su dosímetro, y dado que no se dispone de precedentes investigados en la instalación, se ha realizado un estudio para dilucidar si las dosis registradas pueden recibirse o no en el desempeño laboral normal de dicho puesto de trabajo.

Durante estos procedimientos el paciente se expone al haz directo de RX, mientras que el resto de personal presente en la sala solo puede exponerse a la radiación dispersada por el paciente y, además, bajo el preceptivo delantal plomado de uso obligado en intervencionismo. De todo este personal presente durante el procedimiento, es el especialista en cardiología intervencionista el que recibe, con diferencia, las dosis mayores de radiación dispersa, ya que suele trabajar a pie de tubo y en contacto directo con el paciente (dispersor), mientras que el personal de enfermería permanece a distancias mayores de entre 1 y 2 metros del mismo.

El dosímetro personal irradiado, al igual de todos los que se utilizan en el hospital, incluye cuatro zonas con cuatro detectores de termoluminiscencia TLD bajo diferentes filtros, cada uno de un material: teflón, cobre, sin filtro y aluminio. Utilizando los cocientes de lecturas entre zonas es posible conocer la calidad o energía efectiva de la irradiación que origina la dosis registrada por el dosímetro. Corrigiendo después dichas lecturas según la energía se determina el equivalente de dosis personal, $\mathrm{Hp}(10)$ y el equivalente de dosis superficial, $\mathrm{Hs}(0.07)$ como estimador de la dosis efectiva y de la dosis superficial recibida por el portador del dosímetro.

Normalmente a partir de dichos cocientes de lecturas se puede conocer la energía efectiva de la irradiación, y saber así si la exposición recibida ha sido en haz directo o haz disperso. En el caso estudiado, sin embargo, las energía efectivas obtenidas fueron diferentes para cada cociente $\mathrm{Cu} / \mathrm{PTFE}, \mathrm{Cu} /$ open, $\mathrm{Al}$ /PTFE y Al/open, por lo que la dosis profunda $\mathrm{Hp}(10)$ asignada de $125.5 \mathrm{mSv}$, corresponde a una energía efectiva de $36 \mathrm{keV}$, obtenida con solo los 2 últimos de estos 4 cocientes. Por lo tanto del análisis del CND no se puede deducir de forma concluyente si el dosímetro se ha irradiado o no en haz directo, o lo que es lo mismo, si la irradiación se ha producido por la radiación directa o dispersa producida por el tubo de RX.

El propósito por lo tanto de este trabajo es evaluar las dosis de radiaión implicadas en estos procedimientos y discernir en que condiciones ha sido irradiado el dosímetro de la auxiliar, bien en su posición normal sobre la solapa del uniforme durante le trabajo habitual, o bien en otras condiciones que puedan ser más plausibles. Para ello se miden con un monitor ambiental los equivalentes de dosis individual profunda, $\mathrm{Hp}(10)$, en diferentes puntos de la sala en torno al equipo, en condiciones que simulan las de los procedimientos reales. Por otra parte se mide el rendimiento del equipo en haz directo, para poder estimar las dosis que se reciben en el mismo.

Una vez caracterizadas y conocidas las posibilidades de irradiación del dosímetro, se contrastan los resultados obtenidos con las lecturas experimentales registradas por el dosímetro irradiado. De dicha comparación se extraerán las conclusiones correspondientes, buscando la posible reproducción del episodio real de irradiación. Sobre todo se estudia la posibilidad de que el dosímetro se haya irradiado en la solapa del trabajador, para cuyo fin esta diseñado, en cuyo caso las lecturas registradas estimarían las dosis efectivas que el auxiliar habría podido recibir en el transcurso de su trabajo. 
Además se realiza la vigilancia médica especializada de la trabajadora con objeto de determinar posibles alteraciones sanguíneas u de otro tipo que irían asociadas a una dosis absorbida del orden de magnitud existente en el dosímetro.

\section{MATERIAL Y MÉTODOS}

\section{Dosímetro personal irradiado}

La descripción detallada del dosímetro personal irradiado con sus 4 cristales de termoluminiscencia (TLD) y los filtros correspondientes incluidos en el dosímetro (Ref ${ }^{1}$ ), se muestran en la figura 1.

Figura 1. Fotografía dosímetro con las 4 zonas y los 4 TLD de FLi

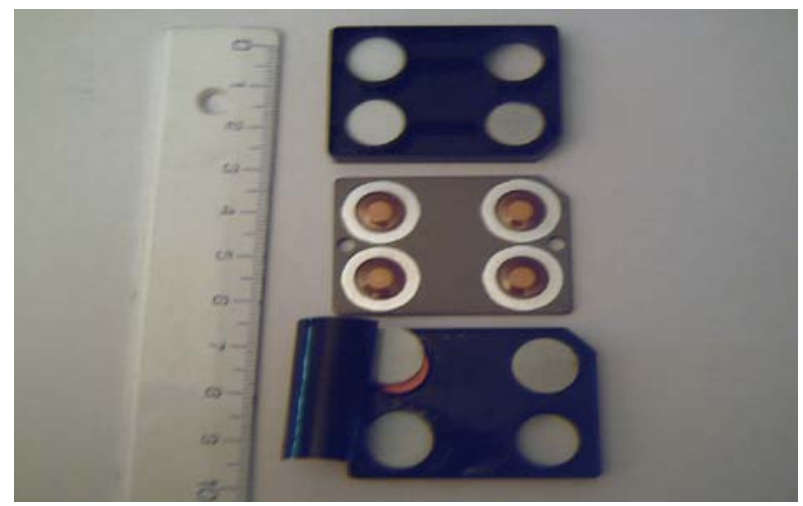

La información correspondiente a las 4 zonas de lectura fue suministrada por el Centro Nacional de Dosimetría, encargado del registro dosimétrico del personal del hospital, y se recoge en la tabla 1:

Tabla 1. Datos del dosímetro irradiado

\subsection{Dosis leídas}

\begin{tabular}{|c|c|c|c|c|c|}
\hline $\begin{array}{l}\text { TLD-100 (FLi) } \\
\text { n. }^{\circ}\end{array}$ & Filtro & $\begin{array}{c}\text { Lecturas corregidas } \\
\text { (u.a.) }\end{array}$ & Нp (10) & $\begin{array}{c}\text { Dosis profunda } \\
(\mathrm{mSv})\end{array}$ & $\begin{array}{c}\text { Dosis } \\
\text { superficial }\end{array}$ \\
\hline 1 & $3.9 \mathrm{~mm}$ PTFE & 75.4 & 117.24 & \multirow{5}{*}{$\begin{array}{c}125.5 \\
\text { (Promedio } \\
\text { de } 1,3 \text { y } 4 \text { ) }\end{array}$} & \multirow{5}{*}{128.1} \\
\hline \multirow{2}{*}{2} & $0.5 \mathrm{~mm}$ PTFE & 8.7 & \multirow{2}{*}{98.21} & & \\
\hline & $3 \mathrm{~mm} \mathrm{Cu}$ & & & & \\
\hline 3 & Open & 102.2 & 130.22 & & \\
\hline 4 & $4 \mathrm{~mm} \mathrm{Al}$ & 53.8 & 128.92 & & \\
\hline
\end{tabular}

1.2 Energías asignadas

\begin{tabular}{lcc}
\hline & Cociente & Eeff (keV) \\
\hline $\mathrm{Al} / \mathrm{ptfe}$ & 0.71 & 40 \\
$\mathrm{Cu} / \mathrm{ptfe}$ & 0.12 & 72 \\
$\mathrm{Al} /$ open & 0.53 & 34 \\
\hline $\mathrm{Cu} /$ open & 0.08 & 69 \\
\hline
\end{tabular}




\section{Procedimientos intervencionistas RFCA/BiVICD}

En el transcurso de un procedimiento diagnóstico con RX en una sala de EF, se producen normalmente 2 situaciones de exposición:

1. en el haz directo al que solo se expone el paciente objeto del procedimiento.

2. en el haz disperso producido por el mismo paciente y al que pueden exponerse los profesionales sanitarios que llevan a cabo el procedimiento, sobre todo y de forma significativa el cardiólogo responsable.

Se trabaja siempre con el tubo de RX bajo el paciente dirigido hacia la zona del corazón del paciente.

\section{Medidas experimentales del tubo emisor de RX}

El tubo emisor de RX de la sala estudiada es un equipo SIEMENS POLYMAT 100, nominalmente de $100 \mathrm{kVp}$, con intensidades variables de 1 a $4 \mathrm{~mA}$, con exposímetro automático, por lo que ajusta, en función del espesor del paciente, el KVp y la intensidad. El rango de estos parámetros así como el rendimiento del equipo determinado recientemente ( $\operatorname{Ref}^{2}$ ) a $70 \mathrm{~cm}$. del foco durante la revisión anual de control de calidad del equipo, se presentan en la tabla 2.

Figura 2. Fotografía del monitor ambiental usado en las medidas de dosis

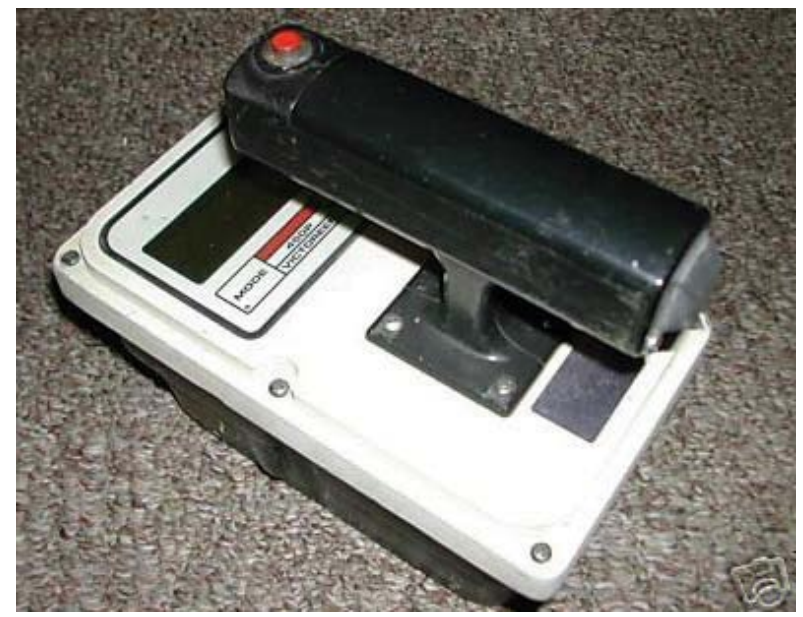

\section{Medidas experimentales de dosis dispersa en la sala durante los procedimientos con monitor ambiental}

Se simula el paciente mediante un dispersor de agua colocado en la posición del mismo sobre la camilla. Durante la irradiación, la intensidad registrada por el equipo de RX de es de $3.4 \mathrm{~mA}$ y $80 \mathrm{kVp}$. Se efectúan medidas de tasa de equivalente de dosis individual $\mathrm{Hp}(10)$ en diferentes posiciones en torno al dispersor Se utiliza un monitor VICTOREEN 450P n/s 1494 calibrado por el suministrador del mismo, Global Calibration Laboratory. Los valores de tasa de dosis medidos se incluyen en la tabla 4 .

Por otra parte las medidas de la tabla 4 están realizadas con un único dispersor, siendo el espesor de los pacientes, así como su peso o su IMC, ambos muy variables entre pacientes e influyendo notablemente en la dosis dispersa. De hecho algunos autores correlacionan las dosis en intervencionismo con el producto del tiempo de escopia por el peso o por el IMC de cada paciente $\mathrm{f}^{4}$. Este hecho se tuvo en cuenta al escoger un dispersor lo suficientemente grueso para representar las condiciones más desfavorables, es decir las de mayor dispersión, correspondientemente leídas en el equipo como de $96 \mathrm{kV}$ y $3.4 \mathrm{~mA}$, (ver tabla de rendimientos del equipo en la tabla 2). 


\section{RESULTADOS}

\section{Dosímetro personal irradiado}

Como puede verse en la tabla 1 en el dosímetro irradiado hay energías muy diferentes. Por estudios realizados mediante Monte Carlo (Ref ${ }^{3}$ ), energías tan elevadas y tan bajas como las presentes en el dosímetro irradiado, de $34 \mathrm{keV}$ a $72 \mathrm{keV}$, solo están presentes en el haz de RX recién emitido por el foco, obteniéndose un coeficiente de variación entre las energías detectadas del $30 \%$. A medida que el haz se va degradando y aumenta la componente dispersa las energías se van homogeneizando, disminuyendo el coeficiente de variación a valores inferiores al $20 \%$. Los espectros de radiación dispersa son más homogéneos con menos picos y con una energía casi constante en torno a 50-60 keV.

\section{Dosis directa producida por el tubo emisor de RX}

Tabla 2. Rendimiento y parámetros experimentales del equipo de RX determinados a $70 \mathrm{~cm}$ del foco emisor

\begin{tabular}{ccccc}
\hline $\mathbf{K V} \mathbf{p}$ & $\mathbf{m A}$ & $\mathbf{R}(\mathbf{m G y} / \mathbf{m i n})$ & Paciente simulado con & $\begin{array}{c}\text { Tamaño de campo (diámetro) } \\
\mathbf{a} \mathbf{7 0} \mathbf{~ c m ~ d e l ~ f o c o ~}\end{array}$ \\
\hline 71 & 1.9 & 13.62 & & 27 \\
80 & 2.4 & 21.3 & $2 \mathrm{~mm} \mathrm{de} \mathrm{Cu}$ & 17 \\
81 & 2.4 & 21.78 & & 14 \\
60 & 1.4 & 7.14 & $1 \mathrm{~mm} \mathrm{de} \mathrm{Cu}$ & 27 \\
67 & 1.7 & 11.58 & & 17 \\
69 & 1.8 & 11.76 & $2 \mathrm{~mm} \mathrm{de} \mathrm{Cu+1} \mathrm{mm} \mathrm{de} \mathrm{Pb}$ & 14 \\
110 & 4.1 & 63.54 & & 27 \\
\hline
\end{tabular}

El rango de valores medidos a $70 \mathrm{~cm}$ concuerda bien con los medidos por Taylor JB et al (Ref ${ }^{4}$ ) para la dosis en piel de pacientes sometidos a procedimientos RFCA y BiVICD, con un valor medio de $32 \mathrm{uSv} / \mathrm{min}$ un máximo de $81 \mathrm{mGy} / \mathrm{min}$ y un mínimo de $25 \mathrm{mGy} / \mathrm{min}$.

Si corregimos los valores de la tabla, medidos a $70 \mathrm{~cm}$ con el factor correspondiente al cuadrado de la distancia, resulta que la tasa de exposición a 1 metro del tubo emisor va de 6 a $32 \mathrm{mGy} / \mathrm{min}$, pudendo suponerse como representativo de las condiciones de trabajo el promedio de estos, $10.6 \mathrm{mGy} / \mathrm{min}$.

\section{Dosis dispersa medida en la sala}

En la tabla 3 se presentan los resultados de las medidas efectuadas en la sala de electrofisiología con el monitor VICTOREEN 450P y simulando el paciente mediante un dispersor de agua colocado en la posición del mismo.

Tabla 3. Medidas ambientales de radiación dispersa en la sala

\begin{tabular}{lcc}
\hline Descripción punto de medida & $\begin{array}{c}\text { Distancia al eje del haz } \\
\text { de } \mathbf{R X}(\mathbf{c m})\end{array}$ & microSv/min \\
\hline Craneal & 50 & 57 \\
Lateral izquierdo del paciente & 50 & 65 \\
Lateral derecho del paciente & 50 & 60 \\
Caudal & 50 & 65 \\
Craneal & 100 & 20 \\
Lateral izquierdo del paciente & 100 & 20 \\
Lateral derecho del paciente & 100 & 23 \\
Caudal & 100 & 20 \\
Entre craneal y lateral izquierdo & 200 & 5 \\
Entre caudal y lateral izquierdo & 200 & 5 \\
\hline
\end{tabular}




\section{DISCUSIÓN Y CONCLUSIONES}

Del análisis de energías realizado sobre las lecturas en los 4 TLD del dosímetro personal irradiado (ver tabla 1) se deduce, aunque no de forma concluyente, que es más probable que la irradiación se haya producido en haz directo que en haz disperso.

Los valores medidos con monitor ambiental de la tabla 4, representan las tasas de dosis a recibir por las distintas personas que ocupan la sala aparte del paciente durante posprocedimientos. La distancia de $50 \mathrm{~cm}$ es representativa de la que corresponde a la posición del intervencionista, sin delantal plomado Las distancias de 100-200 cm. son más representativas de las que corresponden a las posiciones del auxiliar, también sin delantal plomado. Como lo que nos ocupa es estimar dosis reales en dosímetro personal de la auxiliar, se cogerán como más representativos de la dosis máxima a recibir sin delantal plomado, el valor medio de las cuatro medidas realizadas a $100 \mathrm{~cm}$ y reflejadas en las filas 6 a 9 de la tabla 4, es decir $20.75 \mathrm{microSv} / \mathrm{min}$.

Estos valores nos permiten estimar el tiempo de escopia necesario para acumular una dosis como la registrada en el dosímetro de la auxiliar en su posición habitual de trabajo y sin delantal plomado, que resulta ser de 6048 minutos (12500 uSv divididos por el promedio medido a $100 \mathrm{~cm}, 20.75 \mathrm{uSv} / \mathrm{min}$ ). Este tiempo es mucho mayor en realidad, ya que se debe considerar la protección de $0.5 \mathrm{~mm}$ de plomo del delantal de protección que siempre usan todos los profesionales que participan en la intervención, y que se estima que reduce las dosis en un factor 30, (Ref. 4). O sea que en realidad los tiempos anteriores se deberían multiplicar por 30, resultando ser 181445 minutos o 3024 horas.

Este valor resultan ser 168 veces el tiempo real de escopia utilizado el mes en estudio, que resultó ser de 1069 minutos (18 horas), ya que el equipo de RX almacena este dato de forma automática y es facilmente recuperable.

En la tabla 4 siguiente se analizan los tiempos necesarios para alcanzar las dosis registradas por cada dosímetro individual en función de la posición de cada persona con y sin delantal plomado en la sala.

Tabla 4. Análisis de tiempos necesarios para producir las dosis medidas en el dosímetro personal irradiado según la posición en la sala

\begin{tabular}{|c|c|c|c|c|}
\hline \multirow[t]{2}{*}{$\mathbf{P}^{\mathbf{o}}$} & \multirow{2}{*}{ Escenario de irradiación: } & \multicolumn{2}{|c|}{$\begin{array}{l}\text { Tiempo necesario para tener } \\
\qquad \mathrm{Hp}(10)=125 \mathrm{mSv}\end{array}$} & \multirow{2}{*}{ Ocupado por: } \\
\hline & & minutos & horas & \\
\hline 1 & $\begin{array}{l}\text { Radiación dispersa a } 100 \mathrm{~cm} \text { del eje del haz } \\
\text { de RX sin delantal plomado }\end{array}$ & 6048 & 101 & electrofisiólogo \\
\hline $\begin{array}{c}1 \\
\mathrm{pb}\end{array}$ & $\begin{array}{l}\text { Radiación dispersa a } 100 \mathrm{~cm} \text { del eje del haz } \\
\text { de RX con delantal plomado de } 0.5 \mathrm{~mm}\end{array}$ & 181446 & 3024 & electrofisiólogo \\
\hline 2 & $\begin{array}{l}\text { Radiación dispersa a } 50 \mathrm{~cm} \text { del eje del haz } \\
\text { de RX sin delantal plomado }\end{array}$ & 2510 & 42 & auxiliar \\
\hline $\begin{array}{c}2 \\
\mathrm{pb}\end{array}$ & $\begin{array}{l}\text { Radiación dispersa a } 50 \mathrm{~cm} \text { del eje del haz } \\
\text { de RX con delantal plomado de } 0.5 \mathrm{~mm}\end{array}$ & 75300 & 1255 & auxiliar \\
\hline 3 & Directa $80 \mathrm{kV}$ a 1 metro & 12 & 0.2 & paciente \\
\hline
\end{tabular}

En la última fila se ha incluido la dosis en piel del paciente, según el valor obtenido arriba a 1 metro del foco en haz direct0o, $10.6 \mathrm{mSv} / \mathrm{min}$.

Como puede verse solo se obtienen tiempos inferiores a 18 horas, que es el de uso del equipo de RX durante el mes de la irradiación, en la última fila de la tabla 4, es decir para radiación directa, (0.12 horas o 12 minutos). A la vista del valor obtenido, de 12 minutos, se concluye que si el dosímetro personal con $125 \mathrm{mSv}$, se hubiera irradiado en 
haz directo a una distancia de unos $30 \mathrm{~cm}$ del foco de RX, el tiempo resultante sería de de un minuto, aproximadamente.

Esta posibilidad también fue manifestada por la trabajadora, quien narro que en cierta ocasión el dosímetro se le había caído sobre el tubo de RX durante una emisión en escopia un periodo de tiempo muy pequeño. Teniendo en cuenta que el tubo de RX siempre se coloca apuntando al techo, la ventana de salida del haz queda aproximadamente a 33 centímetros del foco emisor y en posición horizontal, por lo que si el dosímetro se cayó y permaneció en esta ventana de salida del haz durante un minuto habría recibido, precisamente, $125 \mathrm{mSv}$.

Se concluye que fue el dosímetro y no la auxiliar quien se expuso, durante un minuto, aproximadamente, al haz directo de RX a una distancia de $33 \mathrm{~cm}$ del foco emisor. La irradiación se produjo al caer el dosímetro sobre la ventana del tubo de RX colocado hacia el techo, permaneciendo durante dicho minuto allí mientras el tubo emitía RX.

\section{REFERENCIAS BIBLIOGRÁFICAS}

1. Dosímetro personal del Centro Nacional de Dosimetría.

2. Cámara de medida del kerma en aire para estimar el rendimiento.

3. R. Barquero, Cálculo Monte Carlo de espectros en una sala de Radiodiagnóstico. Comunicación presentada en el Simposium Internacional sobre Protección del paciente, Málaga, octubre 2006.

4. J. B. Taylor, K. A. Selzman, An evaluation of fluoroscopic times and peak skin doses during radiofrecuency catheter ablation and biv internal cardioverter defibrillator implant procedures, Health Physics Journal 96(2):138-143; 2009.

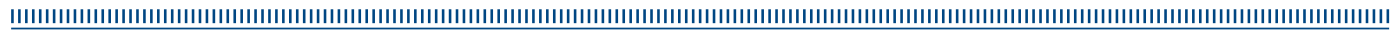

